\title{
Carbon-Nanotube-Deposited Long Period Fiber Grating for Continuous Refractive Index Sensor Applications
}

\author{
Y. C. Tan, W. B. Ji, V. Mamidala, K. K. Chow*, and S. C. Tjin
}

\author{
School of Electrical and Electronic Engineering \\ Nanyang Technological University \\ 50 Nanyang Avenue, Singapore 639798 \\ *E-mail: kkchow@ntu.edu.sg
}

\begin{abstract}
We present a carbon-nanotube-deposited long period fiber grating for refractive index sensing applications in liquid. Carbon nanotubes are deposited around the surface of a long period fiber grating to form the refractive index sensing element. The sensing mechanism relies mainly on the high refractive index properties of the carbon nanotube thin film, which enhances the cladding mode of the long period fiber grating in order to have a significant interaction between the propagating light and the target medium. A sensitivity of $31 \mathrm{~dB} / \mathrm{RIU}$ and $47 \mathrm{~dB} / \mathrm{RIU}$ are obtained for the refractive index ranges of 1.33 to 1.38 and 1.38 to 1.42 , respectively, which have not been demonstrated with normal long period fiber gratings as the sensing element. As the sensing mechanism is based on the change of the transmitted optical power, our proposed scheme can intrinsically solve the limitations of the free spectral range commonly seen in other reported schemes, and continuous and repeatable measurements can be obtained while only acquiring errors mainly from the power fluctuations from the light source. The fiber grating also does not require any further mechanical modification like etching or tapering, which allows the sensing
\end{abstract}


element to have the advantage of mechanical strength for practical applications. The experimental results are consistent with the modeling of the sensing mechanism.

Keywords: optical fiber sensor; carbon nanotube; refractive index sensor; optical materials

\section{$\underline{\text { Introduction }}$}

Optical fiber based refractive index (RI) sensors have attracted a lot of research attention over the years due to the advantage of remote and real-time monitoring capabilities [1-13]. In particular, detecting of RI variations in water is important for water treatment plants as different RI values would indicate the purity level of the water being treated. A variety of optical fiber based sensing schemes such as multimode interference [10], fiber tapering [3, 5, 8, 12], surface plasmon resonance (SPR) [1, 13], and dielectric multilayer films [7, 9] have been previously reported to construct the sensing probe in order to detect RI variations. However, most of these schemes like tapering, which involves the stretching of the fiber to very thin diameters, and SPR, which commonly involves the etching of the fiber cladding, lead to the modification of the fiber structure to enhance the sensitivity, but make the sensing element mechanically weak and hence prone to damage in practical applications [2, 12, 13]. Also, some of the proposed schemes rely on the measurement of interferometic wavelength shifts with RI variation within a certain spectral range. These fiber sensors, though extremely sensitive to external perturbations and immune to power fluctuations as long as a certain extinction ratio is achieved, are usually limited by a certain free spectral range (FSR) of the transmission or reflection spectrum $[5,8,12]$. This poses a considerable challenge when measurements over a wide range of refractive indices are needed. 
In order to meet the conditions of a robust fiber sensor, a sensing element using a long period fiber grating (LPFG) is one of the solutions as it does not affect the mechanical strength of the fiber while providing interaction between the propagating light and the outside medium through the cladding mode of the LPFG [14]. However, one major limitation of such a RI sensing scheme is that the LPFGs only display a significant response to ambient RI changes for RI values close to that of the fiber cladding's RI and very little variation in wavelength for RI values near that of pure water of around $1.33[15,16,17]$. Such low sensitivity at the RI of 1.33 makes the conventional LPFG unsuitable as a RI sensor for applications for water quality monitoring. Previous reports show that the assembling or specific coating of thin films on the LPFGs can enhance the RI sensing range, sensitivity, or specificity [18-23]. However, most of these methods require very precise fabrication techniques with long and complex fabrication processes [18-23].

In this paper, we propose and demonstrate a novel RI sensing scheme using LPFG with carbon nanotubes (CNTs) deposited on the LPFG grating region as the sensing element. CNT is known to be a dark material with high absorption of light and high RI [24]. On the other hand, it was found that CNTs are highly compatible to silica and able to form thin films on silica surfaces $[25,26]$. In our experiment, a relatively simple and effective deposition method was used to coat a layer of CNT around the grating region of the LPFG, making the overall sensor easily reproducible and requiring little mechanical modifications of the fiber structure. Furthermore, due to its sensing principle, the sensor was able to register variations over a range of refractive indices (RIs) and was also not limited by the FSR of its spectrum during the detection of perturbations in its external environment. The sensor also demonstrated good 
repeatability and a continuous RI sensing with a sensitivity of $31 \mathrm{~dB} / \mathrm{RIU}$ and $47 \mathrm{~dB} /$ RIU obtained within the ranges of 1.33 to 1.38 and 1.38 to 1.42 , respectively.

\section{Principle of RI sensing using CNT-deposited LPFG}

The working principle of RI sensing using CNT-deposited LPFG is based on the light extraction of propagating cladding modes of the LPFG by the deposited CNTs. As light propagates through a LPFG, the fundamental core mode (LP01) would be coupled with the co-propagating cladding modes (LP0m). This coupling occurs when the following phase matching condition is satisfied [27]:

$$
\beta_{01}-\beta_{\text {clad }}^{m}=\frac{2 \pi}{\Lambda}, m=2,3,4 \ldots
$$

where $\beta_{01}$ and $\beta_{\text {clad }}^{m}$ are the propagation constants of the fundamental mode and the $m^{\text {th }}$ co-propagating cladding mode, respectively, and $\Lambda$ is the grating period. Due to the high attenuation of the cladding modes, distinct attenuation bands centered at discrete resonant wavelengths in the transmission spectrum can be observed. These resonant wavelengths can be described by the relation:

$$
\lambda_{m, \text { res }}=\left[\mathrm{n}_{\text {eff }, \text { core }}{ }^{01}\left(\lambda_{m, \text { res }}, \mathrm{n}_{1}, \mathrm{n}_{2}\right)-\mathrm{n}_{\text {eff }, \text { clad }}{ }^{0 m}\left(\lambda_{m, \text { res }}, \mathrm{n}_{2}, \mathrm{n}_{3}\right)\right] \Lambda
$$

where $\lambda_{m, r e s}$ is the resonant wavelength due to the coupling between the fundamental core mode and the $m^{\text {th }}$ cladding mode. $\mathrm{n}_{\text {eff, core }}{ }^{01}$ and $\mathrm{n}_{\text {eff }, \text { clad }}{ }^{m}$ are the effective RIs of both the fundamental core mode and the $m^{\text {th }}$ cladding mode, respectively. $\mathrm{n}_{1}, \mathrm{n}_{2}$ and $\mathrm{n}_{3}$ are the core RI, cladding RI and the RI of the surrounding medium, respectively. Consider the case where the refractive index of the surrounding medium does not exceed that of the cladding. According to equation (2), with the increase of the RI of the surrounding medium, the effective index of the cladding will increase while the 
effective index of the core remains the same. This results in a blue shift of the resonant wavelengths with respect to the increase in the surrounding RI.

However, when the RI of the surrounding medium is higher than that of the cladding, the phase matching condition will no longer be satisfied. The surrounding medium of higher RI than the fiber cladding will result in a loss of the total internal reflection condition of the light guided by the cladding of the LPFG. These guided cladding modes will then behave as radiation modes (leaky cladding modes). A portion of the cladding modes will be reflected at the cladding/external medium interface while the rest will be transmitted and lost to the surrounding medium. The amount of reflectance, which affects the attenuation of the attenuation band at the resonant wavelength, will vary based on the Fresnel reflection coefficients $[14,16]$. Thus far, the treatment of the system can be understood to be that of the LPFG being surrounded by a medium of infinite thickness.

If an additional thin film with higher RI than that of the cladding of the LPFG and of finite thickness is coated on the LPFG, the phase matching condition of equation (1) will again not be satisfied. In our scheme, CNTs are deposited on the surrounding of the grating region of a LPFG to form a thin film. By this approach, the system can be modified to incorporate an additional dielectric layer of finite thickness between the cladding and the surrounding medium of infinite thickness as shown in Fig 1. The system can then be modeled as a four layer cylindrical waveguide where the coupledmode equations can be solved through the calculation of the LP modes, crosscoupling coefficients, and self-coupling coefficients to determine the transmission spectrum [19]. The power of each mode can be expressed as:

$$
P_{0 j}=\frac{\beta_{o j}}{2 \omega \mu_{0}} \int_{r=0}^{2 \pi} d \phi \int_{r=0}^{\eta} R_{0 j}^{2}(\mathrm{r}) r d r, j=1,2,3 \ldots
$$


where $\beta_{0 j}$ is the propagation constant of the $\mathrm{LP}_{0 j}$ mode, $\mu_{0}$ is the permeability of free space, $\omega=k_{0} c$, where $c$ is the speed of light in vacuum, $R(\mathrm{r})$ is the radial variation of the modal field, and $\phi$ is the azimuthal angle.

The behavior of the LPFG would be similar to the case of a LPFG surrounded by a medium of higher RI, and a portion of the power of the cladding mode will be reflected by the CNT layer while the rest will be transmitted [14]. The amount of reflectance of the cladding mode at the fiber cladding/thin film interface can be determined by [28]:

$$
R=\left|\frac{r_{23}+r_{34} e^{-i \tilde{k}_{f l l m}}}{1+r_{23} r_{34} e^{-i \tilde{k}_{f l m}}}\right|^{2}
$$

with

$$
\begin{gathered}
r_{2,3}=\frac{n_{\text {clad }}-\tilde{n}_{\text {film }}}{n_{\text {clad }}+\tilde{n}_{\text {film }}} \\
r_{3,4}=\frac{\tilde{n}_{\text {film }}-n_{\text {ext }}}{\tilde{n}_{\text {film }}+n_{\text {ext }}} \\
\tilde{k}_{\text {film }}=\frac{4 \pi \tilde{n}_{\text {film }} d_{\text {flim }}}{\lambda}=\frac{4 \pi n d_{\text {film }}}{\lambda}-i \frac{4 \pi k d_{\text {film }}}{\lambda}=\beta_{\text {film }}-i a d_{\text {film }}
\end{gathered}
$$

where $a=4 \pi k / \lambda$ is the absorption coefficient of the thin film, $\tilde{n}_{\text {film }}=n-i k, n_{\text {clad }}$ and $n_{\text {ext }}$ are the RIs of the cladding and the surrounding medium, respectively. $r_{x, y}$ in equations (5) and (6) is the amplitude reflection coefficient of the interface between layer $x$ and layer $y$ as shown in Fig 1. From equations (4), (5), (6), and (7), it can be seen that $R$ would vary with $n_{\text {ext }}$. This leads to a variation in the amount of attenuation of the cladding modes, which would result in a change in the attenuation depth of the attenuation band. Therefore, the sensing of the RI of the external 
surrounding medium can be obtained by monitoring the amount of attenuation in the attenuation bands of the LPFG.

\section{Experiment on RI sensing using CNT-deposited LPFG}

In our experiment, a piece of LPFG with a grating length of $2.6 \mathrm{~cm}$ and a grating period of $320 \mu \mathrm{m}$ was adopted. It was fabricated by the amplitude mask technique with a Krypton Fluoride laser at a peak wavelength of $248 \mathrm{~nm}$ and left to anneal at $80{ }^{\circ} \mathrm{C}$ for 24 hours. The deposition of the CNTs on the LPFG was done by spraying the well-dispersed CNT solution. At first CNT solution was prepared by dispersing CNT powder in Dimethylformamide (DMF) solution and sonicating in a water bath. After which, a mechanical spray coating method was employed to evenly coat a CNT layer onto the entire surface of the grating region of the fabricated LPFG. The fabrication cost of the sensor includes the standard procedures of the LPFG fabrication and the spray coating of the CNTs. The spray coating method is known to be cost effective as it only involves the cold transfer of nano-materials while no heat chamber is required [25]. Figs. 2(a) and 2(b) show the optical microscopic images of the bare and CNT-deposited LPFG, respectively. Both images were recorded using the same magnification for comparison. It can be observed that a thin layer of CNT is formed over the grating region of the LPFG, darkening the entire coated region. Although it is difficult to directly measure the thickness of the CNT layer on the $\underline{\text { LPG, it can be estimated through concurrently carrying out the same spray coating of CNTs }}$ on a silicon substrate side by side. Hence, the thickness of the CNT layer deposited on the $\underline{\text { silicon substrate can be estimated through scanning electron microscopy (SEM). In this case, }}$ the thickness of the CNT layer was estimated to be several micrometers.

The experimental setup for RI sensing using the fabricated CNT-deposited LPFG is shown in Fig. 3. Two single-mode fiber cords were spliced to each end of the LPFG. The output of a 
white light source (Yokogawa AQ4305), which delivers an average power of -31.4 dBm over the detecting range of 1000 to $1600 \mathrm{~nm}$, was coupled into one end of the fiber and propagated through the LPFG. The spectrum of the transmitted light was measured using an optical spectrum analyzer (OSA, Ando AQ6317B) with a resolution of $2.0 \mathrm{~nm}$ at the other end of the LPFG.

Fig. 4 shows the transmission spectrum of the CNT-deposited LPFG. In this experiment the attenuation band corresponding to the resonance wavelength at $1463.2 \mathrm{~nm}$ with the largest power attenuation was chosen. This resonant wavelength was chosen for the external medium RI measurements as it is expected to display the largest response to external perturbations within 1.33 (pure water) and 1.44 (fiber cladding) [19]. In our experiment, a power change of around $2 \mathrm{~dB}$ of the resonant wavelength was also observed upon deposition. Note that no additional power compensation was necessary for the CNT deposited LPFG as the power measurements were still obtainable within the power sensitivity of the OSA.

The main test solutions used in the experiment was prepared by dissolving sugar in a fixed amount of de-ionized water and stirred till saturated sucrose solution was obtained. The choice of using sucrose solutions over salt solutions is due to the fact that a wider RI range is obtainable with sucrose solutions than with salt solutions. The saturated sucrose solution was then diluted with different volumes of de-ionized water to form different sucrose solutions of varying RI. The RI of each solution was subsequently confirmed with a digital refractometer (Kruss DR201-95) with a resolution of 0.0001. In our experiment, the range of the RI solutions used was from 1.3332 to 1.4179 . All RI measurements were carried out in an indoor environment at a constant temperature of $23 \pm 0.1^{\circ} \mathrm{C}$ to avoid any temperature induced errors in the RI measurement. The constant temperature can also ensure that there will be no temperature-induced perturbations to the grating which can affect the output spectrum [29]. During the experiment, the CNT-deposited LPFG was placed in a holder over a glass slide to 
ensure that no strain was placed on the grating region. Approximately $1 \mathrm{ml}$ of each sucrose solution with specific RI was dropped on the sensing region of the CNT-deposited LPFG and the spectral changes were recorded on the OSA. Note that the entire sensing region was completely immersed in the solution in order to get an accurate measurement. The CNTdeposited LPFG was thoroughly rinsed with de-ionized water and left to dry after each RI measurement.

\section{$\underline{\text { Results and Discussion }}$}

Fig. 5 shows the transmission spectra of the resonance band of the LPFG with test solutions of different RIs. It is observed that as the RI of the solution increases, the intensity minima of the attenuation band of the LPFG decreases. This is consistent with the theoretical investigation described in the previous session. Since the cladding modes behave as radiation modes in the presence of the CNT coating, thus the amount of reflectance of the cladding modes at the cladding/ thin film interface would depend on the external RI, as highlighted by equation (4). The sensor is found to be able to display clear, distinct power levels over the range of RIs measured, and register a larger amount of reflectance for the lower RI values and a smaller amount of reflectance at higher RI values. There was also very little deviation in the resonance wavelength of the attenuation band. The slight variation in wavelength observed from each reading is likely due to the strain on the LPFG caused by minute differences in the volume of sugar solution used for each trial. Note that due to the sensing scheme, readings of the whole range of RIs could be taken continuously and no prediction of the relative wavelength shift is required, and hence there is no FSR limitation.

Fig. 6 plots the intensity change of the attenuation band against different RIs of the test solutions. In order to test the repeatability of the sensor, the experiment was performed for both increasing and decreasing of RIs of the test solution. The RI range of the test solution 
used for this experiment was from 1.3330 to 1.4175 . The change in power is plotted relative to the corresponding data point with the lowest RI for each measurement. As described in equation (4), the results show a nonlinear behavior for a change in intensity with $\mathrm{RI}$ which is consisten with the findings in previous reports $[7,10,16]$. A single curve was plotted based on the experimental data for both measurements. The maximum error due to the repeatability for all data points was found to be $0.41 \mathrm{~dB}$, which could be attributed to power fluctuations and measurement errors from the white light source and the OSA, respectively. The results indicate that the sensor has good repeatability and is able to consistently demonstrate a change in intensity with RI, regardless of whether it is increasing or decreasing. In our experiment, the achieved sensitivity was approximately $31 \mathrm{~dB} / \mathrm{RIU}$ within a RI range of 1.33 to 1.38 . For the RI range from 1.38 to 1.42 , the obtained sensitivity was approximately $47 \mathrm{~dB} / \mathrm{RIU}$. We acknowledge that the sensitivity of the sensor is not as high as compared to that of $[10]$, which has achieved a sensitivity of $-110 \mathrm{~dB} / \mathrm{RIU}$ in the most linear region. Possible improvement of the sensitivity includes utilizing gratings with shorter period, which $\underline{\text { would result in higher order resonance bands within the detectable region of } 1000 \text { to } 1600 \mathrm{~nm}}$ on the OSA [16]. These higher order resonance bands would exhibit greater sensitivity to external perturbations in the ambient environment and thus increase the sensitivity of the sensor $[16,19]$. On the other hand, the thickness of the CNT layer would be expected to affect the sensitivity. Previous reports have shown that there is an optimized thickness for which a small change in RI in the surrounding medium would result in a large change in the transmission spectrum of the grating $[18,19]$. Hence, more extensive methods can be used to obtain the optimal thickness for the maximum sensitivity. From equation (2), it can be seen that the CNT coating will not affect the grating period. Hence, the behavior of the sensor towards strain and temperature perturbations would be expected to be similar to that given in $[10,29]$, where a change in temperature of the external medium or a change in the amount of 
strain on the LPFG would cause a shift in the resonant wavelength. As such, a dual parameter $\underline{\text { sensor would be possible as a second environmental perturbation such that temperature or }}$ strain can be measured from the variation in wavelength. Also, since the sensing is based on the intensity change within a narrow wavelength range, an OSA can be substituted by an optical band pass filter centered at corresponding wavelength followed by an optical power meter. Future work will focus on achieving the optimal layer thickness of the CNT thin film to achieve a higher sensitivity, to extend the measurement range of RIs to those above that of the cladding and below that of pure water (1.33), and also to explore possible methods of incorporating this sensor into a sensor array. Also, since the sensing scheme works based on variations in intensity to the ambient RI, it would be necessary to compensate for any power fluctuation due to the instability of the light source. Suitable compensation methods will be explored to give more accurate intensity measurements for this sensor. Finally, the CNTs can also be functionalized to allow particular molecules to bind to its functionalized sites, thus extending its capabilities to analyte specific sensing in the environment which could greatly enhance its role as a pollutant sensor as well [30].

\section{$\underline{\text { Conclusion }}$}

In summary, we have presented a novel carbon nanotube deposited long period fiber grating refractive index sensor with a relatively simple fabrication method. The sensing scheme allows the sensor to have no free spectral range limitation and also exhibit variations in intensity over a large range of RI values below that of the fiber cladding. Such RI sensing is not obtainable with bare LPFGs. Since there is no tapering or etching of fiber involved in the fabrication process of the sensing element, the robustness of the CNT-deposited LPFG is similar to normal single-mode fibers and is thus suitable for practical applications in harsh 
environments. The sensor registered a sensitivity of $31 \mathrm{~dB} /$ RIU and $47 \mathrm{~dB} / \mathrm{RIU}$ at lower and higher RI ranges, respectively.

\section{Acknowledgment:}

This work was partially supported by Academic Research Fund Tier 1 Grant (RG22/10) of Ministry of Education (MOE) and Nanyang Technological University, Singapore. 


\section{References:}

[1] M. Mitsushio, S. Higashi, and M. Higo, "Construction and evaluation of a gold-deposited optical fiber sensor system for measurements of refractive indices of alcohols," Sens. Actuators, A, vol. 111, no. 2-3, pp. 252-259, Mar. 2004.

[2] D. King, W. B. Lyons, C. Flanagan, and E. Lewis, "A multipoint optical fibre sensor system for use in process water systems based on artificial neural network pattern recognition techniques," Sens. Actuators, A, vol. 115, pp. 293302, Sep. 2004.

[3] K. Q. Kieu and M. Mansuripur, "Biconical fiber taper sensors," IEEE Photon. Technol. Lett., vol. 18, no. 21-24, pp. 2239-2241, Nov. 2006.

[4] M. Consales, A. Cutolo, M. Penza, P. Aversa, G. Cassano, M. Giordano, A. Cusano, "Carbon nanotubes coated acoustic and optical VOCs sensors: towards the tailoring of the sensing performances," , IEEE Trans. Nanotechnol. vol. 6, no. 6, pp. 601-612, Nov. 2007.

[5] P. Lu, L. Q. Men, K. Sooley, and Q. Y. Chen, "Tapered fiber Mach-Zehnder interferometer for simultaneous measurement of refractive index and temperature," Appl. Phys. Lett., vol. 94, no. 13, Mar. 2009.

[6] M. Consales, A. Crescitelli, M. Penza, P. Aversa, P. D. Veneri, M. Giordano, A. Cusano, "SWCNT nano-composite optical sensors for VOC and gas trace detection," Sens. Actuators, B, vol. 138, no. 1, pp. 351-361, Apr. 2009.

[7] J. R. Zhao, X. G. Huang, W. X. He, and J. H. Chen, "High-Resolution and temperature-insensitive fiber optic refractive index sensor based on fresnel reflection modulated by Fabry-Perot interference," J. Lightw. Technol., vol. 28, no. 19, pp. 2799-2803, Oct. 2010. 
[8] P. F. Wang, G. Brambilla, M. Ding, Y. Semenova, Q. Wu, and G. Farrell, "High-sensitivity, evanescent field refractometric sensor based on a tapered, multimode fiber interference," Opt. Lett., vol. 36, no. 12, pp. 2233-2235, Jun. 2011.

[9] K. S. Kim, Y. Mizuno, M. Nakano, S. Onoda, and K. Nakamura, "Refractive index sensor for liquids and solids using dielectric multilayer films deposited on optical fiber end surface," IEEE Photon. Technol. Lett., vol. 23, no. 20, pp. 1472-1474, Oct. 2011.

[10] S. Silva, O. Frazão, J. L. Santos, and F. X. Malcata, "A reflective optical fiber refractometer based on multimode interference," Sens. Actuators, B, vol. 161, no. 1, pp. 88-92, Jan. 2012.

[11] L. H. Chen, T. Li, C. C. Chan, R. Menon, P. Balamurali, M. Shaillender, B. Neu, X. M. Ang, P. Zu, W. C. Wong, K. C. Leong "Chitosan based fiber-optic Fabry-Perot humidity sensor," Sens. Actuators, B, vol. 169, pp. 167-172, Jul. 2012.

[12] W. B. Ji, H. H. Liu, S. C. Tjin, K. K. Chow, and A. Lim, "Ultrahigh Sensitivity Refractive Index Sensor Based on Optical Microfiber," IEEE, Photon. Technol. Lett., vol. 24, no. 20, pp. 1872-1874, Oct. 2012.

[13] H. Tu, T. Sun, and K. T. Grattan, "SPR-based optical fiber sensors using goldsilver alloy particles as the active sensing material," IEEE Sensors J., vol. 13, no. 6, pp. 2192-2199, Jun. 2013.

[14] D. B. Stegall and T. Erdogan, "Leaky cladding mode propagation in longperiod fiber grating devices," IEEE Photon. Technol. Lett., vol. 11, no. 3 pp. 343-345, Mar. 1999. 
[15] V. Bhatia and A. M. Vengsarkar, "Optical fiber long-period grating sensors," Opt. Lett., vol. 21, no. 9, pp. 692-694, May. 1996.

[16] H. J. Patrick, A. Kersey, and F. Bucholtz, "Analysis of the response of long period fiber gratings to external index of refraction," J. Lightw. Technol., vol. 16, no. 19, pp. 1606-1612, Sep. 1998.

[17] M. Smietana, J. Szmidt, M. L. Korwin-Pawlowski, W. J. Bock, and J. Grabarczyk, "Application of diamond-like carbon films in optical fibre sensors based on long-period gratings," Diamond Relat. Mater., vol. 16, no. 4-7, pp. 1374-1377, Apr. 2007.

[18] N. D. Rees, S. W. James, R. P. Tatam, and G. J. Ashwell, "Optical fiber longperiod gratings with Langmuir-Blodgett thin-film overlays," Opt. Lett., vol. 27, no. 9, pp. 686-688, May. 2002.

[19] I. Del Villar, I. R. Matias, F. J. Arregui, and P. Lalanne, "Optimization of sensitivity in long period fiber gratings with overlay deposition," Opt. Express, vol. 13, no. 1, pp. 56-69, Jan. 2005.

[20] K. M. Tan, C. M. Tay, S. C. Tjin, C. C. Chan, and H. Rahardjo, "High relative humidity measurements using gelatin coated long-period grating sensors," Sens. Actuators, B, vol. 110, no. 2, pp. 335-341, Oct. 2005.

[21] C. C. C. Lam, R. Mandamparambil, T. Sun, K. T. V. Grattan, S. V. Nanukuttan, S. E. Taylor, P. A. Muhammed Basheer, "Optical fiber refractive index sensor for chloride ion monitoring," IEEE Sensors J., vol. 9, no. 5, pp. 525-532, May. 2009.

[22] Q. Li, X. L. Zhang, Y. S. Yu, Y. Qian, W. F. Dong, Y. Li, J. G. Shi, J. T. Yan, H. Y. Wang, "Enhanced sucrose sensing sensitivity of long period fiber 
grating by self-assembled polyelectrolyte multilayers," React. Funct. Polym., vol. 71, no. 3, pp. 335-339, Mar. 2011.

[23] S. Korposh, R. Selyanchyn, W. Yasukochi, S. W. Lee, S. W. James, and R. P. Tatam, "Optical fibre long period grating with a nanoporous coating formed from silica nanoparticles for ammonia sensing in water," Mater. Chem. Phys., vol. 133, no. 2-3, pp. 784-792, Apr. 2012.

[24] V. A. Margulis and E. A. Gaiduk, "Nature of near-infrared absorption in singlewall carbon nanotubes," Phys. Lett. A, vol. 281, no. 1, pp. 52-58, Mar. 2001.

[25] S. Y. Set, H. Yaguchi, Y. Tanaka, and M. Jablonski, 'Laser mode locking using a saturable absorber incorporating carbon nanotubes," J. Lightw. Technol., vol. 22, no. 1, pp. $51-56$, Jan. 2004.

[26] K. K. Chow, S. Yamashita, and S. Y. Set, "Four-wave-mixing-based wavelength conversion using a single-walled carbon-nanotube-deposited planar lightwave circuit waveguide," Opt. Lett. vol. 35, no. 12, pp. 2070-2072 2010.

[27] A. M. Vengsarkar, P. J. Lemaire, J. B. Judkins, V. Bhatia, T. Erdogan, and J. E. Sipe, "Long-period fiber gratings as band-rejection filters," J. Lightw. Technol., vol. 14, no. 1, pp. 58-65, Jan. 1996.

[28] H. A. Macleod, Thin-film optical filters/ H. Anglus Macleod, 4th ed. Boca Raton, FL: CRC Press/Taylor \& Francis, c2010., 2010.

[29] X. W. Shu, Z. Lin, and I. Bennion, "Sensitivity characteristics of long-period fiber gratings," J. Lightw. Technol., vol. 20, no. 2, pp. 255-266, Feb. 2002.

[30] H. Lee, G. Shaker, K. Naishadham, S. Xiaojuan, M. McKinley, B. Wagner, and M. Tentzeris, "Carbon-nanotube loaded antenna-based ammonia gas 
sensor," IEEE Trans. Microw. Theory Techn., vol. 59, no. 10, pp. 2665-2673, Oct. 2011. 


\section{Figure captions:}

Figure 1. Schematic illustration of a carbon nanotube (CNT) deposited long period fiber grating (LPFG).

Figure 2. Optical microscopic images of (a) bare LPFG and (b) LPFG coated with CNT thin film, under the same magnification.

Figure 3. Experimental setup of refractive index (RI) sensing using a CNTdeposited LPFG.

Figure 4. Transmission spectrum of the CNT-deposited LPFG in air.

Figure 5. Output spectra of the resonance band of the CNT-deposited LPFG with different RI of the test solutions.

Figure 6. Plot of the intensity change of the resonance band against RI of the test solution. 


\section{External Medium (layer 4)}

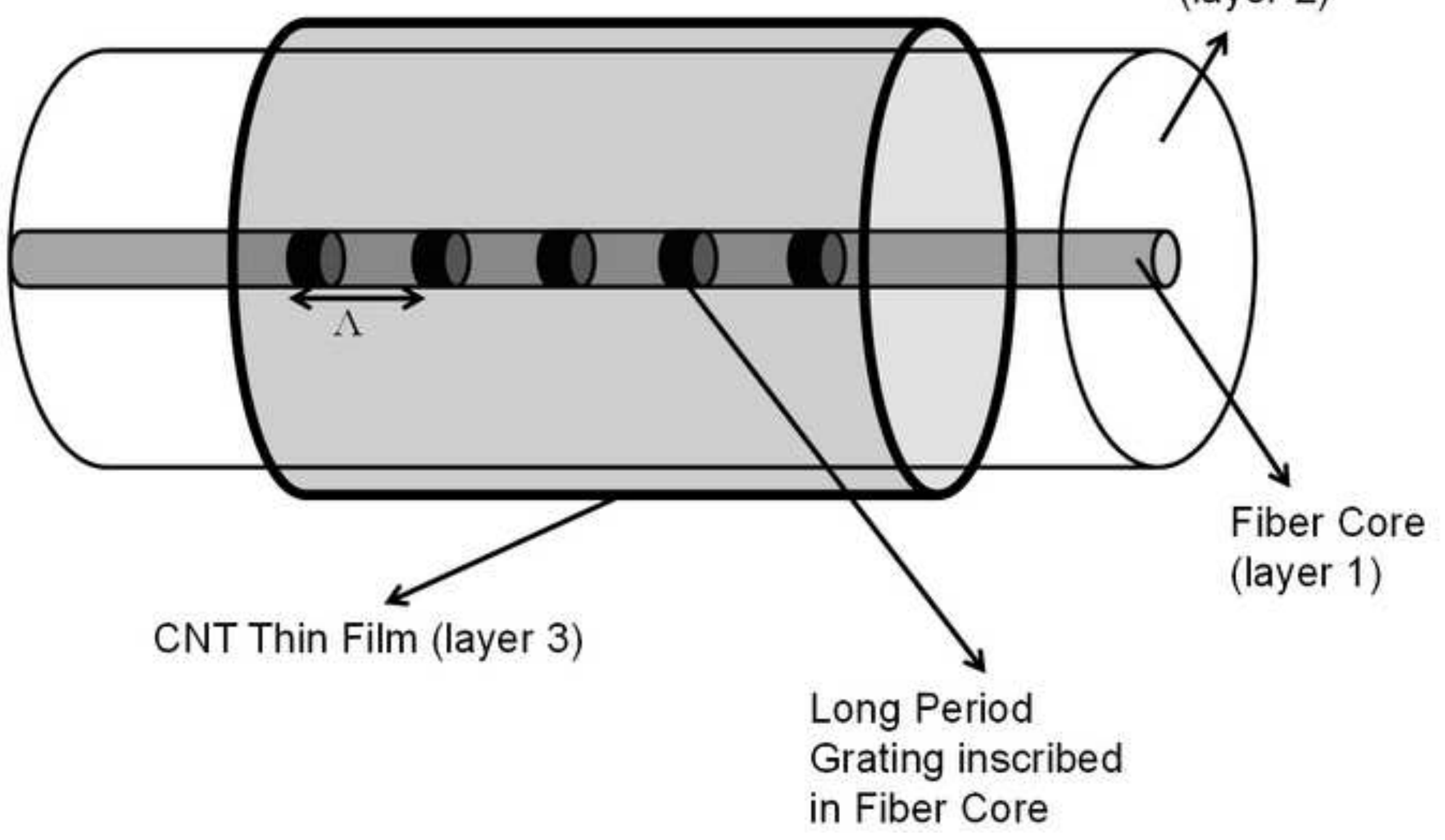

Fiber Cladding (layer 2)
Fiber Core (layer 1) 


\section{Figure(s)}

Click here to download high resolution image

(a)

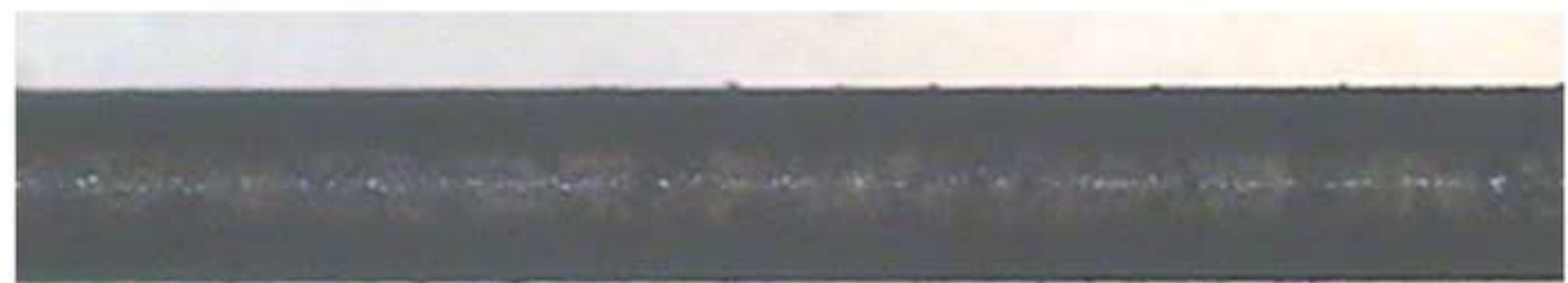

(b) 


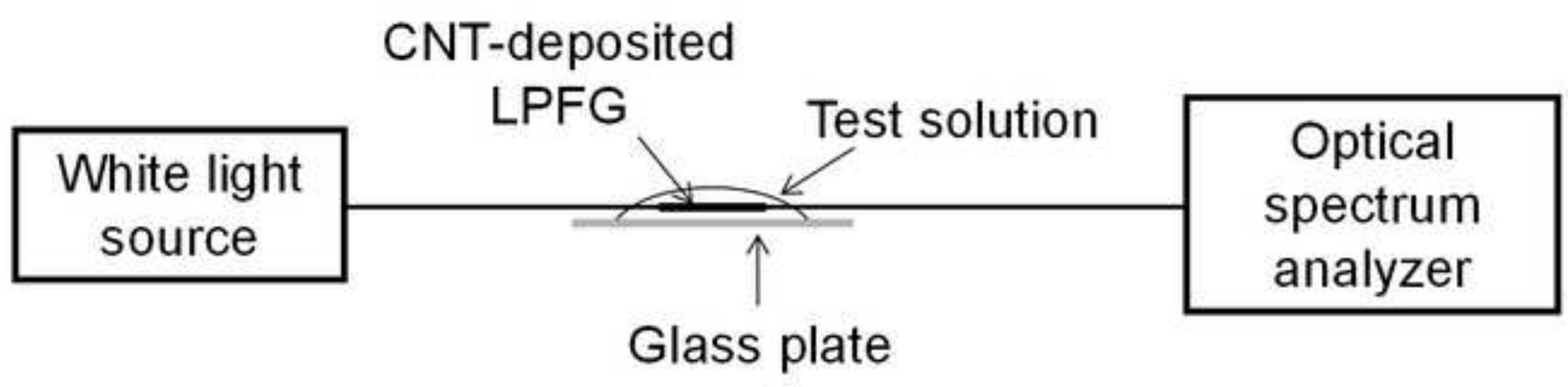


Click here to download high resolution image

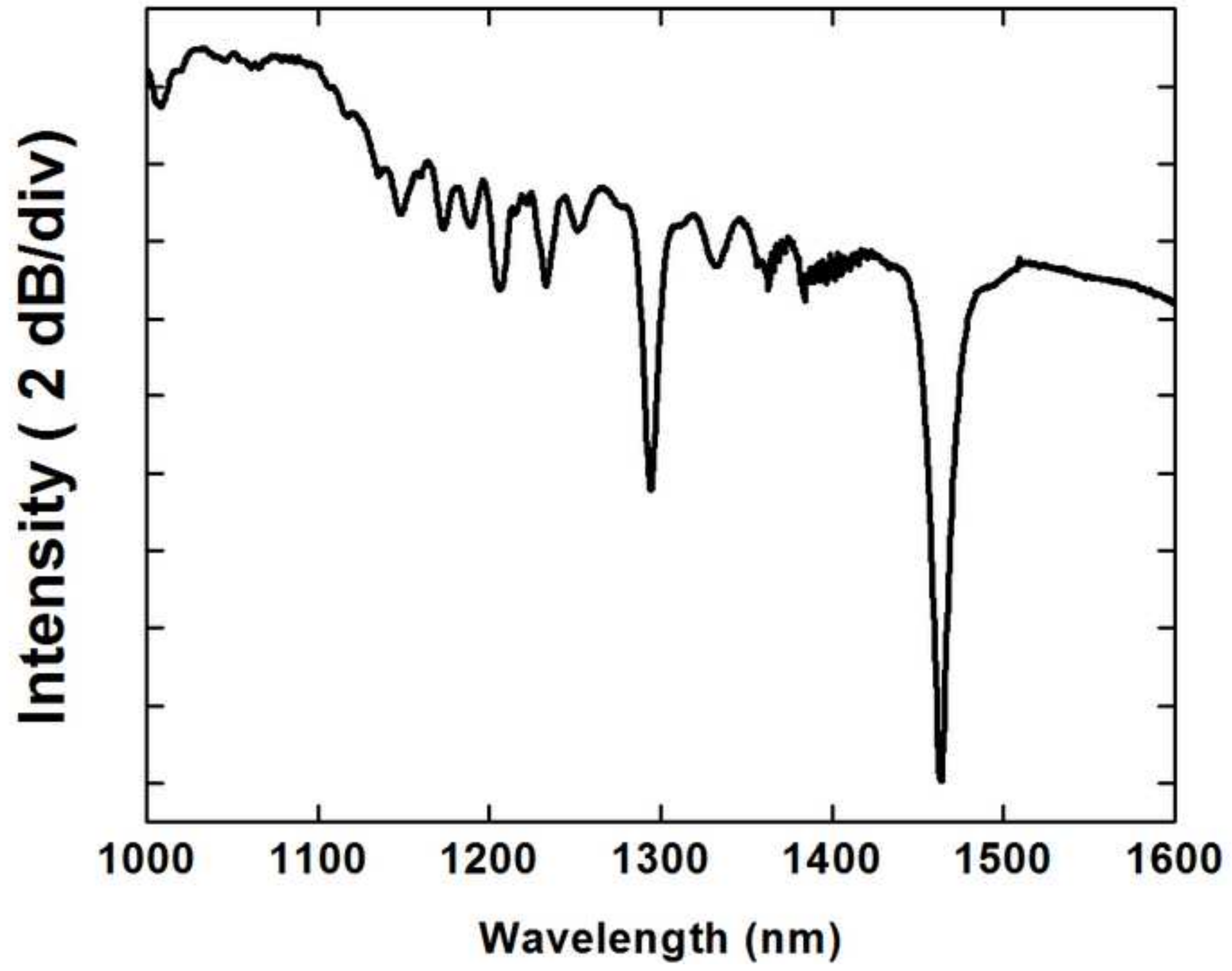




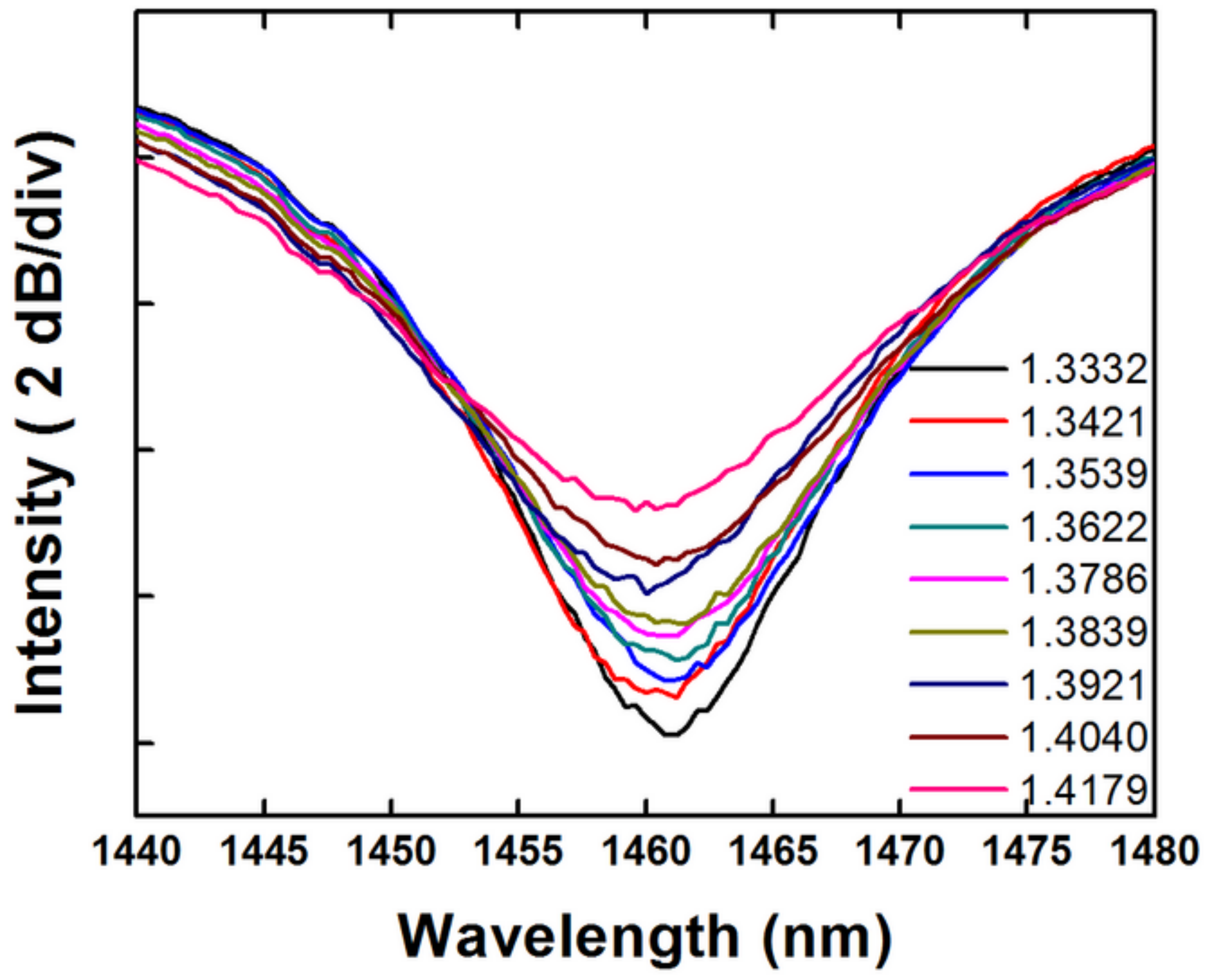




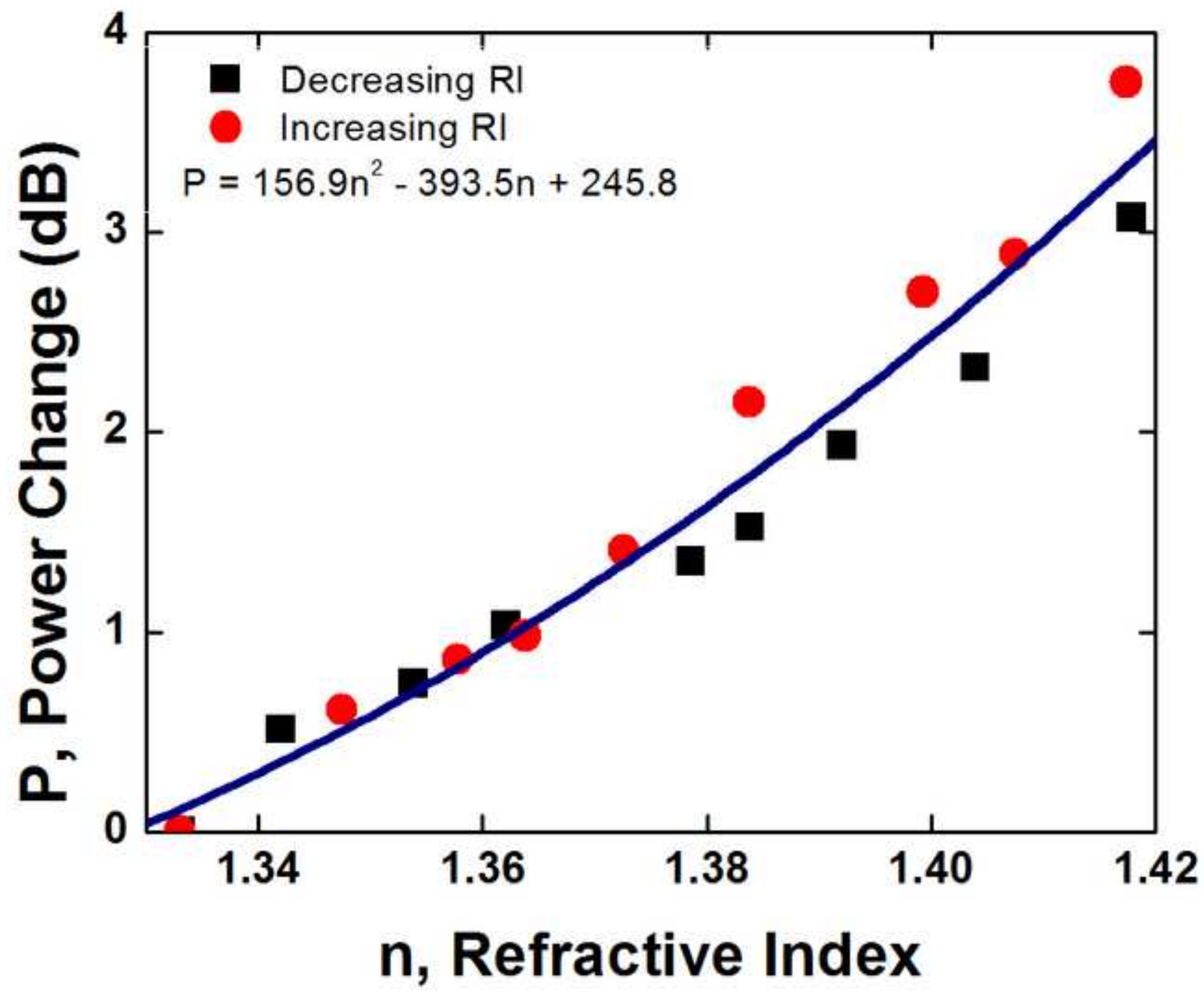

